\title{
Patients with haemolytic uraemic syndrome caused by Escherichia coli O157: Absence of antibodies to Vero cytotoxin 1 (VT1) or VT2
}

\author{
H Chart, D Law, B Rowe, D W K Acheson
}

\begin{abstract}
Serum samples from 30 patients with haemolytic uraemic syndrome (HUS), caused by Escherichia coli O157, and 30 apparently healthy volunteers, were used to examine the immune response of patients to Vero cytotoxins (VT) 1 and VT2. Patients' sera could not be differentiated from control sera using ELISA; and using immunoblotting, none of the sera had antibodies reactive with either the $A$ or $B$ subunits of VT1 or VT2. Examination of sera for antibodies to VT1 and VT2 seems to be of little value in the serodiagnosis of HUS caused by Vero cytotoxin-producing $\boldsymbol{E}$ coli 0157 .
\end{abstract}

(f Clin Pathol 1993;46:1053-1054)

Strains of $E$ coli $\mathrm{O} 157: \mathrm{H} 7$ belong to a group of $E$ coli that produce Vero cytotoxins 1 and 2 (VT1 and VT2) and are termed VTEC. ${ }^{1}$ VTEC, and in particular $E$ coli $\mathrm{O} 157: \mathrm{H} 7$, are a cause of haemolytic uraemic syndrome (HUS). Isolating VTEC or detecting VT from patients' faeces can indicate the causative agent of HUS; but VTEC and VTs are present in patients' faeces for only a short period of time after onset of infection, and therefore the causal agent may go undetected. ${ }^{2}$ Patients infected with $E$ coli 0157 produce antibodies to the lipopolysaccharide of this organism ${ }^{3}$ and serology has been used to provide evidence of infection. In the present study sera from patients with HUS were examined for serum antibodies to VT1 and VT2 with the aim of improving and extending available serological tests for obtaining evidence of infection with $E$ coli $O 157$ and other VTEC.

\section{Methods}

Sera from 30 patients with HUS, caused by $E$ coli 0157 , and from 30 control children were used. Antisera prepared in rabbits to purified VT1 and VT2 were also used.

Vero cytotoxins 1 and 2 were prepared by receptor analog affinity chromatography with immobilised P1 glycoprotein. ${ }^{4}$ Enzyme linked immunosorbent assay (ELISA) plates were coated with VT1 or VT2 $(0 \cdot 1 \mu \mathrm{g}$ per well in coating buffer) and the sera were diluted in phosphate buffered saline (PBS). ${ }^{3}$ Human antibodies of the IgG and IgM class were assayed for using goat anti-human IgG or IgM immunoglobulins conjugated to alkaline phosphatase (Sigma Chemical Co Ltd), respectively. Both were diluted to manufacturer's specifications in PBS. Rabbit antibodies of the IgG class were assayed for using goat anti-rabbit IgG immunoglobulin conjugated to alkaline phosphatase (Sigma Chemical Co Ltd). All antibody conjugates were diluted to manufacturer's specifications in PBS. Bound antibody conjugates were detected using the enzyme substrate $p$-nitrophenyl phosphate (Sigma Chemical Co Ltd.) and quantified by measuring the absorbance at $405 \mathrm{~nm}\left(\mathrm{~A}_{405}\right)$.

Sodium dodecyl sulphate-polyacrylamide gel electrophoresis (SDS-PAGE) and immunoblotting was carried out as described ${ }^{3}$; using $10 \mu \mathrm{g}$ VT per lane, protein profiles were either stained with Coomassie blue or transferred on to nitrocellulose sheets for reaction with sera. Human antibodies of the IgG and IgM class were assayed for using goat anti-human IgG and IgM immunoglobulins labelled with ${ }^{125}$ Iodine. Rabbit antibodies were detected using radioiodinated goat anti-rabbit IgG, $10^{6} \mathrm{cpm} /$ lane. VTantibody complexes were detected by autoradiography.

\section{Results}

Using a range of toxin concentrations, from $0.1 \mu \mathrm{g}$ to $100 \mathrm{pg}$ per well, and a range of rabbit antiserum dilutions, from $\times 100$ to $\times 12800$; an optimal antigen coating concentration of $0 \cdot 1 \mu \mathrm{g}$ VT per well was established for VT1 and VT2. Using this antigen concentration, sera from patients with HUS and controls were reacted with VT1 and VT2 by ELISA. Using serum dilutions of $\times 1000$, patients' and control sera gave mean ELISA values of $<0.5\left(\mathrm{~A}_{405}\right)$. ELISA values obtained from patients' sera were not significantly different from those obtained with control sera $(p=0.005)$. Diluting sera 100 -fold resulted in higher ELISA values over all, but patients' sera could not be differentiated from control sera.

Protein profiles of VT1 contained bands of 31,27 , and 10 kilodaltons designated $A, \star$, and $B$ in the figure (lane 1). SDS-PAGE profiles of VT2 contained bands of 34, 27, and 11 kilodaltons given the same designations (lane 2). Reacting SDS-PAGE profiles of VT1 with a rabbit-anti VT1 antiserum showed that this antiserum contained specific antibodies which reacted with bands of 31 , 27 , and 10 kilodaltons (lane 3 ) but not with VT2 (lane 4). Rabbit-anti VT2 did not react 


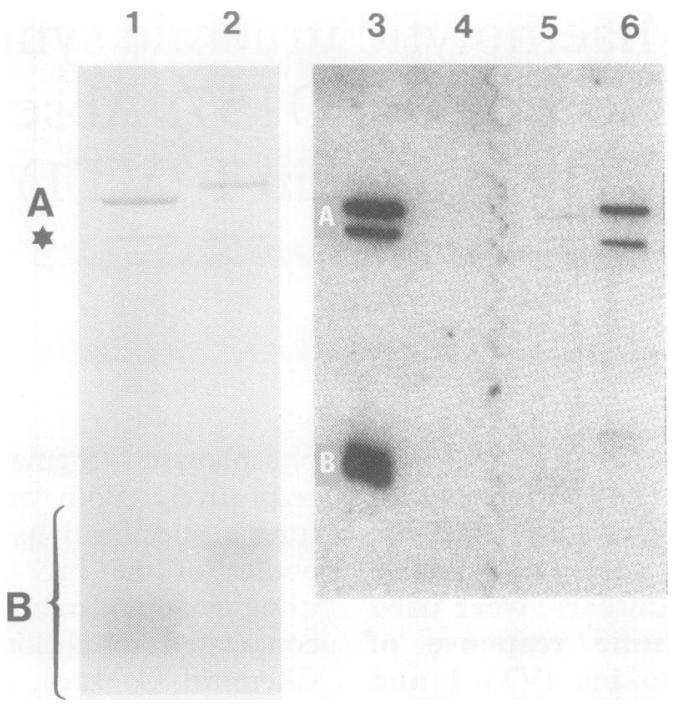

SDS-PAGE profiles of VT1 (lane 1) and VT2 (lane 2) showing bands comprising $A$ and $B$ subunits, and $a$ possible $A_{1}$ fragment of VT1 and VT2 ( $\star$ ). Rabbit antibodies produced to VT1 reacted with $A, B$ and $\star$ bands of VT1 (lane 3) but not VT2 (lane 4). Similarly, antibodies produced to VT2 did not react with VT1 lane 5) but reacted with $A, B$ and $\star$ bands of VT2 (lane 6).

$10 \mu \mathrm{g}$ of toxin was used per lane.

with bands in profiles of VT1 (lane 5) but did react with bands of 34, 27, and 11 kilodaltons in profiles of VT2 (lane 6). Patients' sera did not react with either VT1 or VT2 by immunoblotting.

\section{Discussion}

ELISA and immunoblotting techniques have been used to show that patients with HUS produce antibodies to the lipopolysaccharide of $E$ coli 0157 . Using these techniques, we examined sera from patients with HUS, caused by $E$ coli 0157 , for antibodies to preparations of VT1 and VT2. The 31 and 10 kilodalton bands observed in profiles of VT1, after SDS-PAGE, were thought to comprise the 32 kilodalton $A$ subunit and the $7 \cdot 7$ kilodalton B subunit of VT1, as reported by Levin et al. ${ }^{5}$ The 27 kilodalton protein band in profiles of VT1 were thought to be the $A_{1}$ subunit of the $A$ portion of the holotoxin, a theory supported by the observed reaction of the 27 kilodalton band with a rabbit antiserum to VT1. Similarly, the 34 and 11 kilodalton bands detected in profiles of VT2 were also considered A and B subunits of VT2, respectively, and again the 27 kilodalton protein band was thought to be the $A_{1}$ fragment of the A portion of VT2. From this part of our study, we concluded that VT1 did not share antibody binding sites with VT2. Using ELISA, patients' sera could not be differentiated from control sera. Barrett et $a l^{6}$ examined the sera from patients with
HUS and reported neutralising antibodies to VT; antibodies to VT were also detected in these sera using a capture ELISA. ${ }^{6}$ This same report, however, claimed that antibodies could be detected in sera from both patients and controls, and consequently these workers concluded that serological tests involving serum antibodies specific for VT were insensitive.

In our study we optimised experimental procedures for detecting antibodies to VT1 and VT2 using toxin specific rabbit antisera and highly purified VT1 and VT2. Applying these procedures for the analysis of control and patients' sera specific antibodies to these toxins could not be detected.

VT binds readily to certain human cells via galactose $\alpha$ 1-4 galactose $\beta$ 1-4 glucose ceramide, ${ }^{7}$ suggesting that the amounts of "free" VT in the blood are probably very small, and the possibility arises that the concentrations of VT in patients may be too low to stimulate the immune defence system.

Serological tests, based on antibodies to lipopolysaccharides, have been used to provide evidence of infection with $\mathrm{VTEC}^{8}$ and could be used for routine antibody tests, although the application may be limited. Unfortunately, although VT production is a common characteristic of this group of organisms, our study suggests that serological tests based on antibodies to VT have no diagnostic value for providing evidence of infection with $E$ coli $\mathrm{O} 157$ or other VTEC.

Studies for Vero cytotoxin purification were supported by Public Health Service grants $\mathrm{AI} / 16242$ and $\mathrm{AI} / 20235$ from the National Institute for Allergy and Infectious Diseases, Bethesda, Maryland, USA.

1 Smith HR, Scotland SM. Verocytotoxin-producing strains of Escherichia coli. 7 Med Microbiol 1989;26:77-85.

2 Milford DV, Taylor CM, Gutteridge B, Hall S, Rowe B, Kleanthos $H$. Haemolytic uraemic syndrome in the British Isles, 1985-1988; association with Vero cytoBritish Isles, 1985-1988; association with Vero cytotoxin-producing Escherichia coli. Part 1: clinical and epidemio

3 Chart H, Scotland SM, Rowe B. Serum antibodies to Escherichia coli serotype O157:H7 in patients with hemolytic uremic syndrome. F Clin Microbiol 1989;27: 285-90.

4 Donohue-Rolfe A, Acheson DWK, Kane AV, Keusch GT. Purification of Shiga toxin and Shiga-like toxins I and II by receptor analog affinity chromatography with immobilized P1 glycoprotein and production of crossreactive monoclonal antibodies. Infect Immun 1989;57: 3888-93.

5 Levin ML, Walters MDS, Barret TM. Hemolytic uremic syndrome. Adv Ped Infect Dis 1989;4:51-82.

6 Barrett TJ, Green JH, Griffin PM, Pavia AT, Ostroff SM, Barrett TJ, Green JH, Griffin PM, Pavia AT, Ostroff SM,
Wachsmuth IK. Enzyme-linked immunosorbent assays for detecting antibodies to Shiga-like toxin 1, Shiga-like for detecting antibodies to Shiga-like toxin 1, Shiga-like toxin 2, and Escherichia coli O157:H7 lipopolysacc

7 Lingwood CA, Law H, Richardson SE, et al. Glycolipid binding of natural and recombinant Escherichia coli produced Verotoxin in vitro. $\mathcal{F}$ Biol Chem 1987;262: 8834-9.

8 Chart H, Rowe B. Serological identification of infection by Vero cytotoxin producing Escherichia coli in patient with haemolytic uraemic syndrome. Serodiagnost Immunother Infect Dis 1990;4:413-18. 